\title{
Multiple prostatic abscesses presenting with urethral discharge
}

\author{
S K Gill, R J C Gilson, D Rickards
}

\begin{abstract}
Prostatic abscess has become less common, is now usually related to urinary tract infection, and is a rare cause of urethral discharge. The case is described of a man with prostatic abscesses caused by Staphylococcus aureus possibly related to recent skin abrasions. Transrectal ultrasound was used to make the diagnosis and to facilitate repeated drainage with a successful outcome.
\end{abstract}

\section{Introduction}

Prostatic abscess has become less common, ${ }^{1}$ is difficult to diagnose and its aetiology is often uncertain. In the pre antibiotic era many cases were due to gonococcal or metastatic staphylococcal infections. ${ }^{2-4}$ Recently it has been more frequently associated with urinary tract infections usually with coliform organisms.

The modes of presentation of prostatic abscess are varied and it cannot be reliably diagnosed clinically. The differential diagnosis includes prostatitis, urinary tract infection, benign hypertrophy of the prostate and carcinoma of the prostate or bladder. The diagnosis may be further complicated by masking of the infectious process by antibiotic therapy. ${ }^{256}$ Recently the introduction of transrectal ultrasonography (TRUS) has allowed a more rapid diagnosis together with ultrasound guided aspiration and biopsy for microbiological and histological examination. Drainage can also be established.

In a recent review of 269 cases $^{1}$ in the world literature urethral discharge was a presenting complaint in only $7 \%$ of patients. We describe a case of multiple prostatic abscesses presenting with urethral discharge in a $\mathbf{4 2}$ year old man in which transrectal ultrasound was valuable for diagnosis, therapeutic drainage and subsequent follow-up.

\section{Case report}

A 42 year old heterosexual male attended the gen-

Academic Department of Genito Urinary Medicine, University College \& Middlesex School of Medicine S K Gill, R J C Gilson

Department of Radiology, The Middlesex Hospital, London, UK

D Rickards itourinary clinic complaining of a profuse urethral discharge and dysuria without frequency for 3 days. In addition he described a dull pain in the perineum. His only sexual partner for the last eight years was his wife. His only past history of a sexually transmitted disease was pediculosis pubis 10 years previously. $\mathrm{He}$ had no other genitourinary symptoms. His only relevant recent history was a fall from a ladder sustaining a closed fracture of his left forearm and multiple abrasions, two weeks earlier.

On examination he was afebrile with his left forearm in plaster of Paris. He was noted to have a profuse green/yellow urethral discharge. On rectal examination an enlarged firm very tender prostate with no definable midline sulcus was palpated.

\section{Investigations and treatment}

Urethral smear showed greater than 20 polymorphs per high power field but no evidence of gonorrhoea. The two glass urine test showed a cloudy first specimen and specks in the second. Dipstick testing of the urine was negative for protein and sugar. A urethral culture for Neisseria gonorrhoeae and a urethral swab for chlamydia antigen (Chlamydiazyme, Abbott Laboratories) were negative, as was serological testing for syphilis. Serum alkaline phosphatase, tartrate labile acid phosphatase, and at follow-up, random blood glucose and full blood count were within normal limits. Also a subsequent HIV test was negative.

The symptoms and signs initially suggested a diagnosis of prostatitis and he was started on a 2 week course of oxytetracycline $500 \mathrm{mg}$ qds. In order to exclude other prostatic pathology TRUS of the prostate was performed. Using a $7.5 \mathrm{mHz}$ linear array endoprobe, the examination showed an enlarged prostate with multiple loculated, predominantly echopoor, areas consistent with prostatic abscesses. The largest of these was aspirated under ultrasound control via the perineal route and $10 \mathrm{ml}$ of purulent fluid obtained(fig). Culture of the fluid yielded a heavy growth of Staphylococcus aureus sensitive to penicillin, erythromycin and oxytetracycline.

\section{Subsequent course}

By the time the patient presented for his first transrectal ultrasound (three days after presentation in the genitourinary clinic) his urethral discharge had resolved. The patient underwent weekly therapeutic 


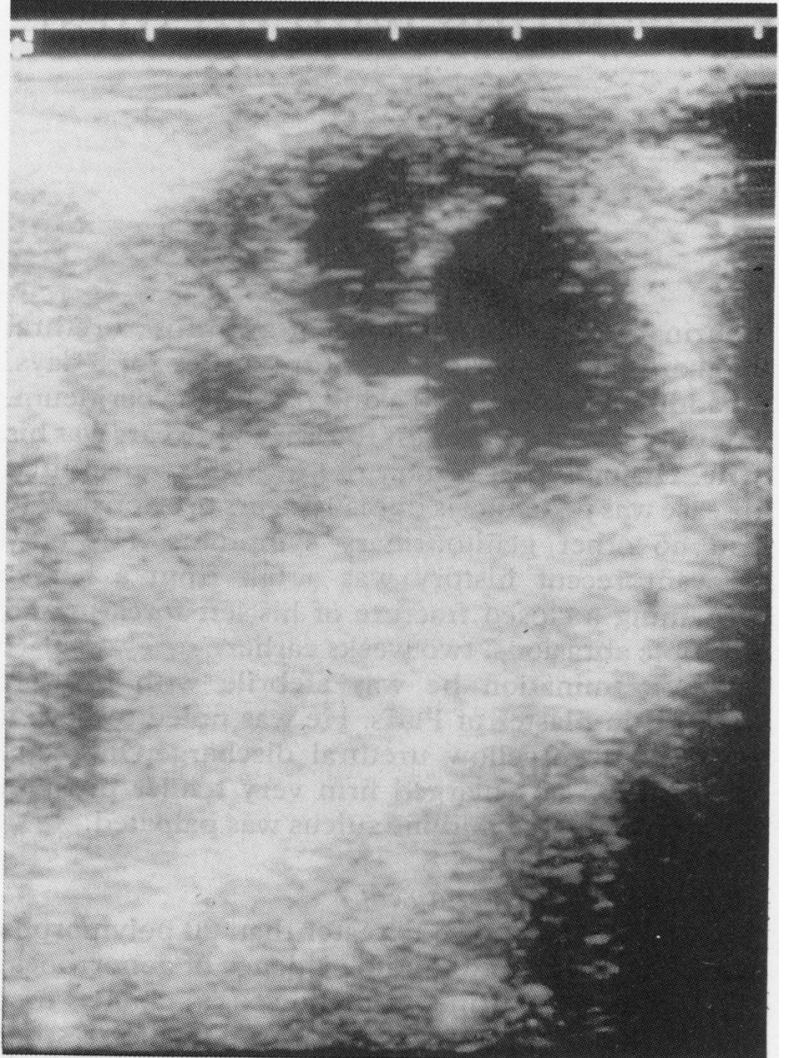

Figure Transrectal ultrasound image: multiloculated abscess (dark, echopoor areas) within the prostate (light, echogenic area).

aspiration of his prostatic abscess (each procedure being covered with gentamicin). He had made a complete clinical recovery one month later and ultrasound showed complete resolution of the abscesses.

\section{Discussion}

This case is unusual in several respects. Firstly, for prostatic abscess to present with urethral discharge is most unusual, occurring in only $7 \%$ of 269 cases reported in the world literature in the last 40 years. ${ }^{1}$ Also given that urethral discharge is a common presenting complaint in a genitourinary clinic, the patient's other symptom of dull perineal pain, which led to the diagnosis, may easily have been overlooked. Secondly, the organism isolated in this patient was Staphylococcus aureus which was identified in only $6 \%$ of 109 cases reviewed in which a microbiological diagnosis had been made. ${ }^{1}$ In the series the most frequent organism isolated was $E$ coli $(20 \%)$ and other gram negative bacilli $(20 \%)$.
It was presumed that the abscess in this case may have occurred as a result of haematogenous spread from the abrasions on the patient's forearm (once the plaster was removed six weeks later there was evidence of healing abrasions). The patient did not have diabetes, HIV infection or evidence of any other cause of immunosuppression. In this case, transrectal ultrasound was valuable both for diagnosis, treatment and follow-up. Other methods used for diagnosis of prostatic abscess have been abdominal ultrasound scanning and CT. ${ }^{78}$ TRUS has emerged as the most sensitive imaging modality for the prostate itself and is also the most appropriate for ultrasound guided aspiration.

With TRUS the appearance of prostatic abscess is characteristic. TRUS allows the differentiation from a diffuse prostatitis, malignancy or benign prostatic hypertrophy. Ultrasound guided aspiration was first described by Weinberger et $a l^{9}$ and enables a microbiological diagnosis to be made and also facilitates therapeutic drainage.

In conclusion, if this symptom complex is seen again in a genitourinary clinic setting it may alert the attendant physician to consider the diagnosis of prostatic abscess. Transrectal ultrasound if available will provide a rapid method of confirming or excluding the diagnosis as well as facilitating subsequent management.

We thank Dr JS Bingham for permission to report this case.

Address for correspondence: Dr RJC Gilson, Academic Department of Genito-Urinary Medicine, James Pringle House, The Middlesex Hospital, London W1A 8AA, UK.

1 Weinberger M, Cytron S, Servadio C, Block C, Rosenfeld JB, Pitlik SL. Prostatic abscess in the antibiotic era. Rev Infect Dis 1988;10:239-49.

2 Youngen R, Mahoney SA, Persky L. Prostatic abscess. Surg Gynecol Obstet 1967;124:1043-6.

3 Sargent JC, Irwin R. Prostatic abscess: a Clinical study of 42 cases. Am J Surg 1931;11:334-7.

4 Bruce AW, Fox M. Acute infections of the prostate gland. $\mathrm{Br} J$ Urol 1960;32:302-5.

5 Becker LE, Harrin WR. Prostatic abscess: a diagnostic and therapeutic approach. $J$ Urol 1964;91:582-5.

6 Persky L, Austen G Jr, Shatten WE. Recent experiences with prostatic abscess. Surg Gynecol Obstet 1955;101:629-33.

7 Sukov RJ, Scardino PT, Sample WF, Winter J, Confer DJ. Computed tomography and transabdominal ultrasound in the evaluation of the prostate. J Comput Assis Tomogr 1977;1: 281-9.

8 Dennis MA, Donohue RE. Computed tomography of prostatic abscess. J Comput Assist Tomogr 1985;9:201-2.

9 Weinberger M, Pitlik SD, Rabinovitz M et al. Per-rectal ultrasonography for diagnosis of and guide to drainage of prostatic abscess (letter). Lancet 1985;ii:772.

Accepted for publication 13 June 1991 